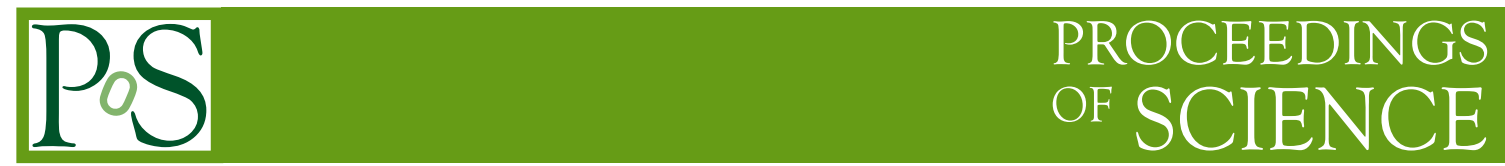

\title{
Measurement of production mode cross sections of the Higgs boson in decays to bosons with the ATLAS detector
}

\section{Liza Mijović ${ }^{a}$ on behalf of the ATLAS Collaboration}

${ }^{a}$ SUPA - School of Physics and Astronomy

University of Edinburgh, United Kingdom

E-mail: liza.mijovic@cern.ch

The ATLAS experiment at the Large Hadron Collider has collected $139 \mathrm{fb}^{-1}$ of proton-proton collision data at centre-of-mass energy of $13 \mathrm{TeV}$. Detailed measurements of Higgs boson properties are being performed with this dataset. This report reviews measurements of the Higgs boson production mode cross sections and simplified template cross sections, using Higgs decays to two photons, two $Z$ bosons and two $W$ bosons.

40th International Conference on High Energy physics - ICHEP2020

July 28 - August 6, 2020

Prague, Czech Republic (virtual meeting) 


\section{Introduction}

The Large Hadron Collider (LHC) has delivered $139 \mathrm{fb}^{-1}$ of proton-proton collisions at the centreof-mass energy of $13 \mathrm{TeV}$ to the ATLAS experiment [1]. This Run 2 data-set corresponds to over one million Higgs boson $(H)$ production events and enables precise cross section measurements in the main production modes: gluon-gluon fusion ( $\mathrm{ggF})$, vector-boson fusion (VBF) and associated production with a vector boson $(V H ; V=W$ or $Z)$, or a top quark pair $(t \bar{t} H)$. The production mode cross sections are measured in the Simplified Template Cross Section (STXS) framework [2] which aims to provide measurements that are easy to combine between different decay channels, have reduced sensitivity to the theory uncertainties as well as high sensitivity to deviations from the Standard Model (SM). To these ends a requirement is made on the Higgs boson rapidity ${ }^{1}$ $\left|y_{H}\right|<2.5$ and the production mode phase-space is partitioned into mutually exclusive regions using event properties such as jet multiplicity or transverse momentum $\left(p_{\mathrm{T}}\right)$ of the Higgs boson. This report summarises the latest such measurements in Higgs decays to two photons $(H \rightarrow \gamma \gamma)$, two $Z$ bosons $\left(H \rightarrow Z Z^{*}\right)$, and two $W$ bosons $\left(H \rightarrow W W^{*}\right)$.

\section{Higgs boson decay to two photons}

The ATLAS collaboration released a new measurement of the STXS production cross sections in the $H \rightarrow \gamma \gamma$ decay mode with $139 \mathrm{fb}^{-1}$ of data [3]. Similar to the previous result [4] the analysis relies on the excellent photon identification and resolution of the ATLAS detector to extract the number of signal (S) events from a narrow signal peak in the di-photon invariant mass distribution $\left(m_{\gamma \gamma}\right)$ shown in Figure 1a. The background (B) is estimated from the $m_{\gamma \gamma}$ side-band in an S+B fit.

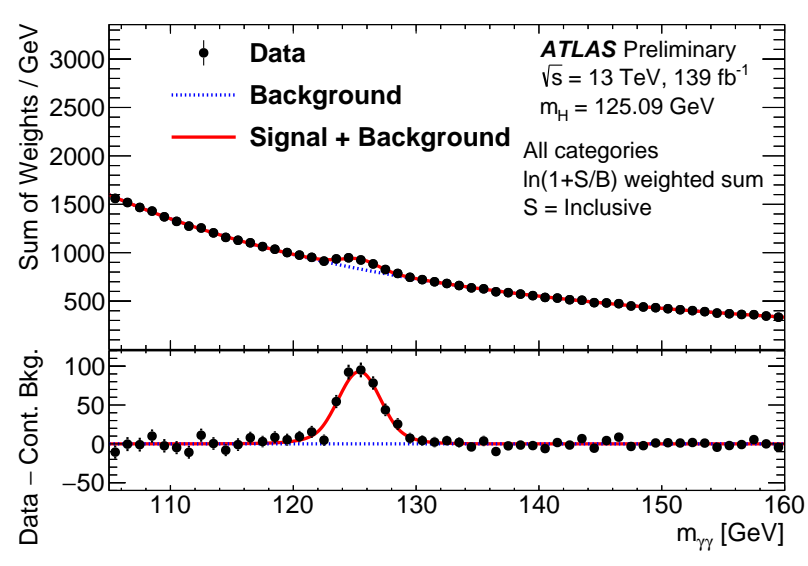

(a)

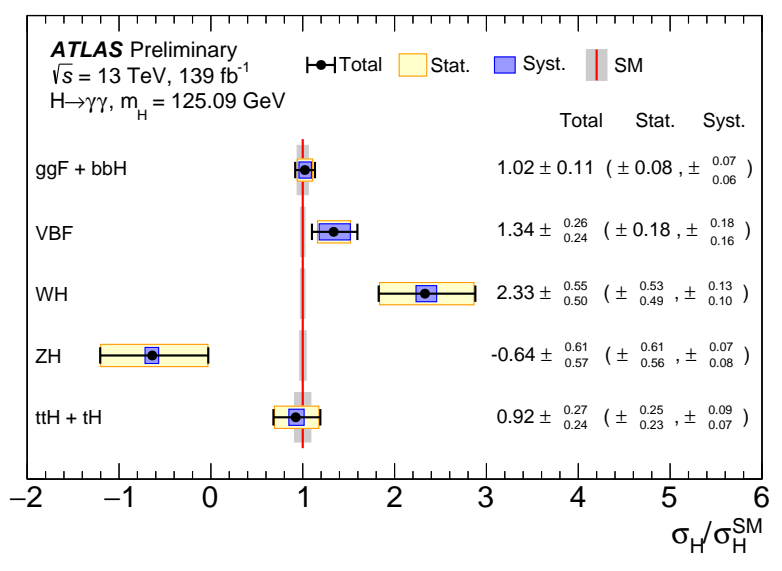

(b)

Figure 1: a) The $m_{\gamma \gamma} \mathrm{S}+\mathrm{B}$ fit to the data and b) production mode cross sections in the $H \rightarrow \gamma \gamma$ decay mode [3].

\footnotetext{
${ }^{1}$ ATLAS uses a right-handed coordinate system with its origin at the nominal interaction point (IP) in the centre of the detector and the $z$-axis along the beam pipe. The $x$-axis points from the IP to the centre of the LHC ring, and the $y$-axis points upward. Cylindrical coordinates $(r, \phi)$ are used in the transverse plane, $\phi$ being the azimuthal angle around the $z$-axis. The pseudorapidity is defined in terms of the polar angle $\theta$ as $\eta=-\ln \tan (\theta / 2)$.
} 


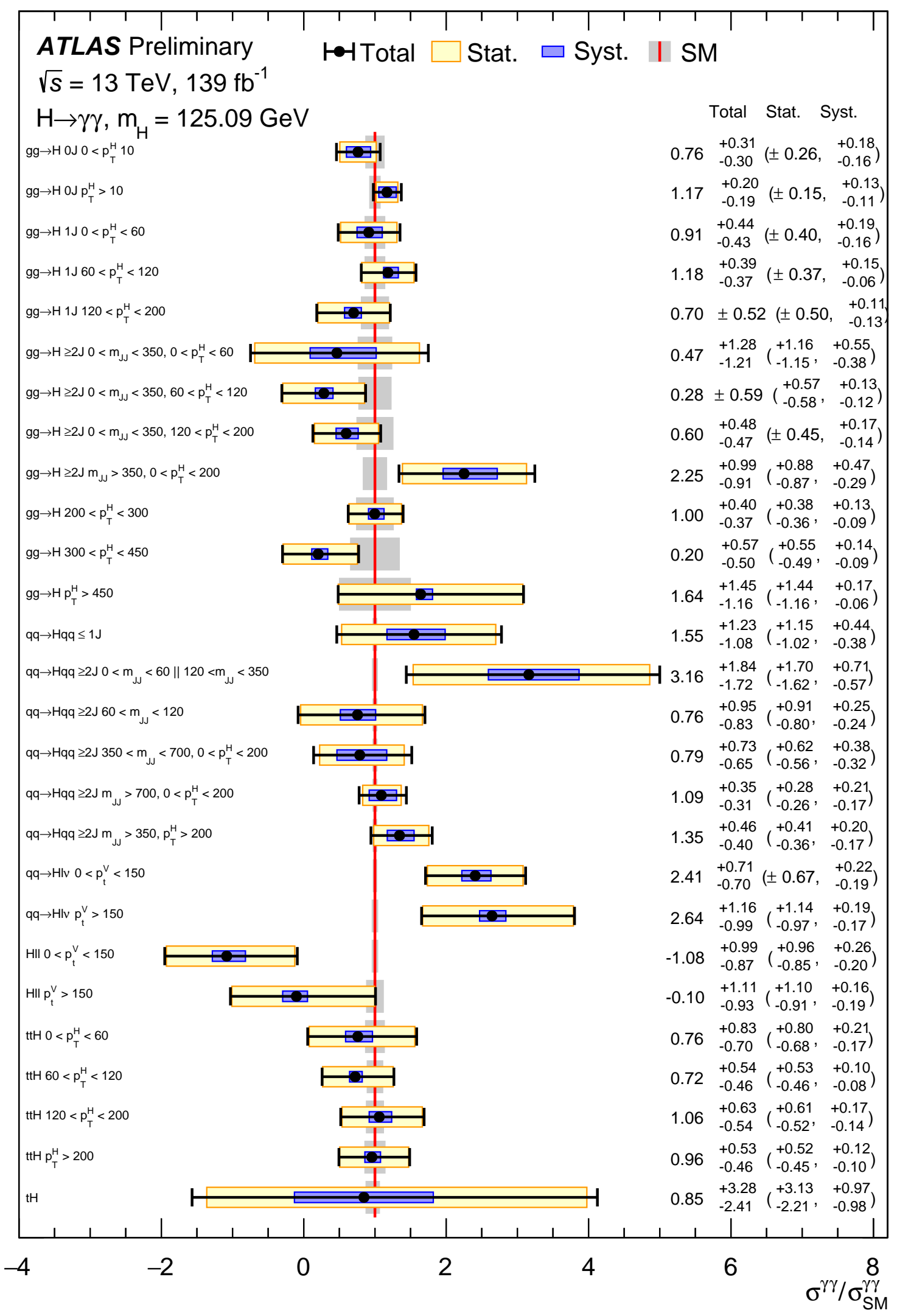

Figure 2: The signal strengths $\left(\sigma / \sigma_{\mathrm{SM}}\right)$ measured in the $27 \mathrm{STXS}$ regions in $H \rightarrow \gamma \gamma$ decays [3]. 
Compared to the previous result the analysis sensitivity is enhanced by a new strategy to classify the signal events into STXS regions and reject the background events. While the previous analyses performed such categorization sequentially, giving higher priority to production processes with lower cross sections, the new strategy considers all production processes simultaneously. It consists of two boosted decision trees (BDT):

- Signal multi-class BDT: splits signal into STXS regions, aiming to simultaneously reduce the uncertainties and correlations between the classes.

- Background rejection binary BDT: in each of the signal multi-class BDT output classes a dedicated BDT is trained. This BDT aims to separate signal from the backgrounds.

The measured production mode cross sections are shown in Figure $1 \mathrm{~b}$. In the ggF and VBF production modes the statistical uncertainty is comparable to the systematic uncertainty. The latter is dominated by the background modelling ( $\mathrm{ggF}$ ) or signal modelling (VBF). The $V H$ and $t \bar{t} H$ production mode cross section measurements are dominated by the statistical uncertainty. The full Run 2 data-set also enables an increased granularity in the number of measured STXS regions compared to the previous result. The analysis measures the cross sections in 27 STXS regions shown in Figure 2. These are found to be compatible with the SM predictions (p-value $=60 \%$ ).

\section{Higgs boson decay to two $W$ bosons}

The ATLAS collaboration released a new measurement of the VBF production cross-section in the $H \rightarrow W W^{*} \rightarrow e v \mu v$ decays with the full Run 2 data-set for this conference [5]. The two highest $p_{\mathrm{T}}$ jets in the event are assumed to stem from the VBF hard process and the events are vetoed in case they contain additional high- $p_{\mathrm{T}}$ jets with pseudo-rapidity in the interval between these two leading jets (central jet veto). The analysis reaches higher sensitivity compared to the previous publication [6]. Besides the larger data-set, this increase is predominantly due to:

- An optimisation of the event selection: the central jet veto is applied for jets with $p_{\mathrm{T}}>30 \mathrm{GeV}$, compared to $p_{\mathrm{T}}>20 \mathrm{GeV}$ used previously, reducing the theory modelling uncertainty.

- A new multi-variate discriminant between the signal and background events. A BDT is replaced by a deep neural network (DNN). The network inputs are optimised and extended.

Most backgrounds in the analysis are taken from simulation, with the exceptions of the backgrounds with top quarks, from $Z+$ jets events and from events with mis-identified leptons, which are estimated from the data control regions (CR). The DNN output is used as the discriminating variable in the statistical analysis, with seven signal region (SR) bins is shown in Figure 3. The bin boundaries are set by requiring at least 10 expected signal and background events per bin as well as a relative statistical uncertainty on the background of $\leq 20 \%$. When these criteria are satisfied, a bin boundary is set once there are more than 20 signal events present in a given bin. The signal over background (S/B) ratio reaches 3.5 in the bin with the highest DNN output. The signal is observed (expected) with a significance of 7.0 (6.2) standard deviations. The measured signal strength is:

$$
\mu_{V B F}=1.04{ }_{-0.12}^{+0.13} \text { stat. }{ }_{-0.08}^{+0.09} \text { exp. }{ }_{-0.12}^{+0.17} \text { sigt. }{ }_{-0.07}^{+0.08} \text { bkgt. }
$$

The stat. and exp. denote the statistical and experimental uncertainty. The sigt. and bkgt. denote the theory modelling uncertainty for each of the signal and background. The signal theory modelling dominates the measurement uncertainty. The sensitivity of this measurement could therefore be enhanced by pursuing a dedicated effort which would reduce the signal modelling uncertainty. 


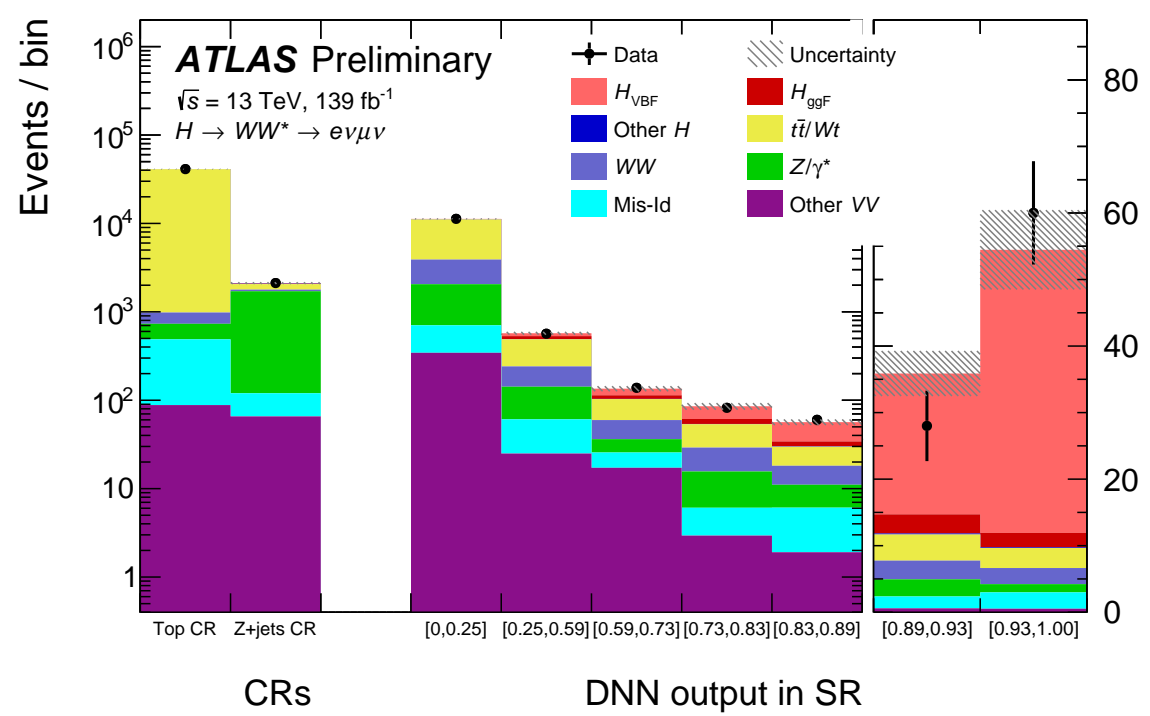

Figure 3: The background control regions and the signal DNN discriminant in $H \rightarrow W W^{*}$ decays [5].

\section{Higgs boson decay to two $Z$ bosons}

The STXS cross sections were measured in the $H \rightarrow Z Z^{*}$ decay mode with $139 \mathrm{fb}^{-1}$ of data in Ref. [7]. The measurement is done in four-lepton decay channel, where lepton $\ell=e, \mu$. This yields about 200 observed (and expected) events. The signal purity is high; it reaches $\mathrm{S} / \mathrm{B} \sim 2$ for events with four-lepton mass close to the Higgs boson mass (Figure 4a). All quantities used to define the the STXS regions, such as jet multiplicity, are found to be well modelled by the simulation. The measured production mode cross sections times the $H \rightarrow 4 \ell$ branching ratio and the signal strengths $\mu=\frac{\sigma \cdot \mathrm{BR}}{\sigma_{\mathrm{SM}} \cdot \mathrm{BR}}$ are shown in Figure $4 \mathrm{~b}$. They are dominated by the statistical uncertainty: $\mu_{\mathrm{ggF}}=0.96 \pm 0.10$ stat. \pm 0.03 exp. ${ }_{-0.03}^{+0.03}$ theo. $\quad \mu_{V H}=1.44_{-0.90}^{+1.13}$ stat. ${ }_{-0.14}^{+0.21}$ exp. ${ }_{-0.17}^{+0.24}$ theo. $\mu_{\mathrm{VBF}}=1.21 \pm 0.44$ stat. ${ }_{-0.08}^{+0.13}$ exp. ${ }_{-0.05}^{+0.07}$ theo. $\quad \mu_{t \bar{t} H}=1.7_{-1.2}^{+1.7}$ stat. \pm 0.2 exp. \pm 0.2 theo.

The cross sections are also measured in 12 STXS regions and found to be consistent with the SM.
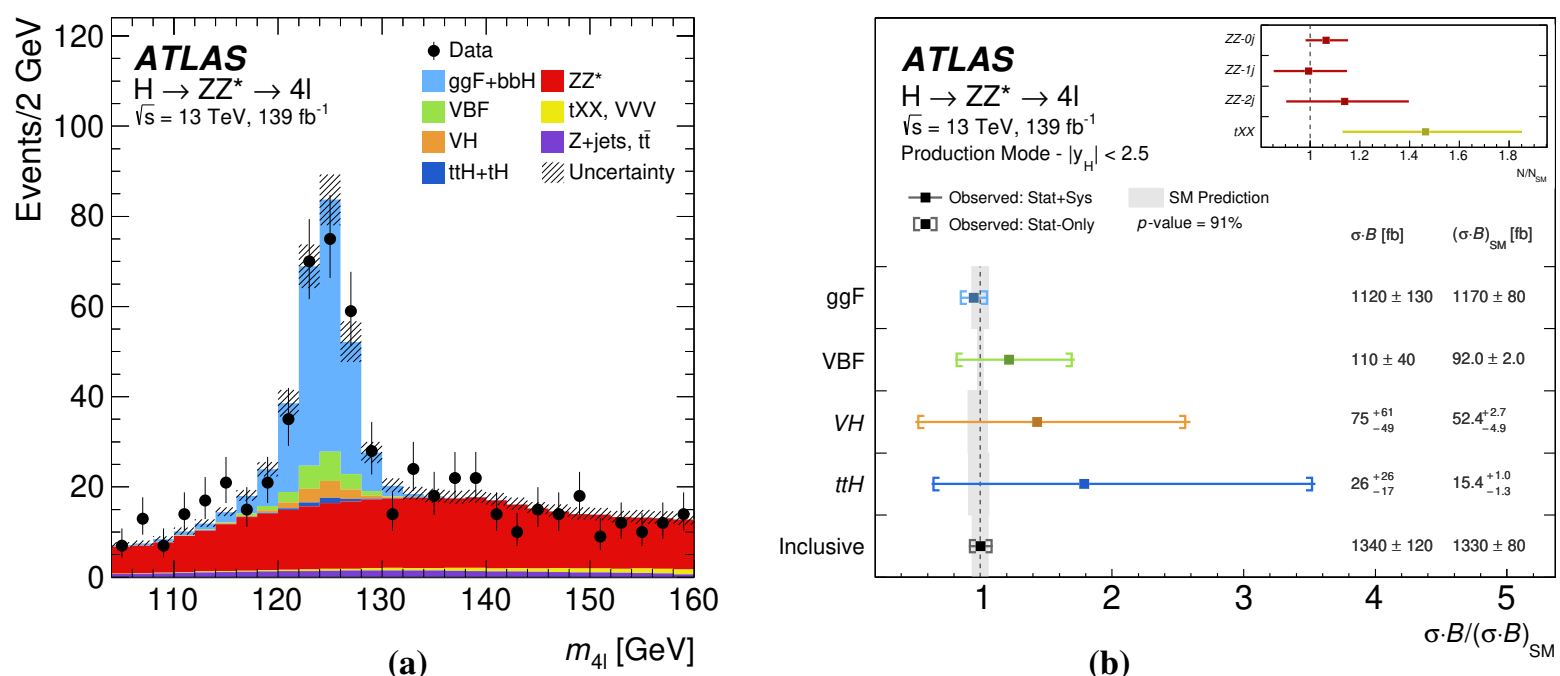

Figure 4: $H \rightarrow 4 \ell$ decay channel: a) Four lepton invariant mass distribution in the data and the simulation. b) The measured production mode cross sections times the branching ratio [7]. 


\section{Summary \& Outlook}

The production mode cross section measurements in Higgs decays to two bosons by the ATLAS collaboration are reviewed. ATLAS released two new preliminary results with the full LHC Run 2 data-set for the ICHEP2020 conference:

- $H \rightarrow \gamma \gamma$ decays: detailed characterisation of the Higgs boson production cross sections in 27 STXS regions. The measurement is compatible with the SM prediction (p-value $=60 \%$ ).

- $H \rightarrow W W^{*}$ decays: observation of the VBF production with a significance of 7.0 (6.2) observed (expected) standard deviations.

Apart from the larger statistics, the largest increase in sensitivity compared to the previous publications comes from the improvements in multivariate discriminants and event selection.

The measurements of the VBF production in $H \rightarrow \gamma \gamma$ and $H \rightarrow W W^{*}$ decays are limited by the signal theory modelling uncertainty. A dedicated effort reducing this uncertainty could therefore increase the sensitivity of the Run 2 measurements.

The measurements in $H \rightarrow Z Z^{*}$ decays and $V H, t \bar{t} H, t H$ production mode measurements in $H \rightarrow \gamma \gamma$ decays remain limited by statistical uncertainty. The sensitivity will therefore increase with the data collected in the future LHC runs.

\section{References}

[1] ATLAS Collaboration, The ATLAS Experiment at the CERN Large Hadron Collider, JINST 3 (2008) S08003.

[2] LHC Higgs Cross Section Working Group, D. de Florian et al., Handbook of LHC Higgs Cross Sections: 4. Deciphering the Nature of the Higgs Sector, arXiv: 1610.07922.

[3] ATLAS Collaboration, Measurement of the properties of Higgs boson production at $\sqrt{s}=13 \mathrm{TeV}$ in the $H \rightarrow \gamma \gamma$ channel using $139 \mathrm{fb}^{-1}$ of pp collision data with the ATLAS experiment, ATLAS-CONF-2020-045 [http://cdsweb.cern.ch/record/2728055] (2020).

[4] ATLAS Collaboration, Measurements of Higgs boson properties in the diphoton decay channel using $80 \mathrm{fb}^{-1}$ of pp collision data at $\sqrt{s}=13 \mathrm{TeV}$ with the ATLAS detector, ATLAS-CONF-2018-028 [http://cdsweb. cern.ch/record/2628771] (2018).

[5] ATLAS Collaboration, Observation of vector-boson-fusion production of Higgs bosons in the $H \rightarrow W W^{*} \rightarrow e v \mu v$ decay channel in pp collisions at $\sqrt{s}=13 \mathrm{TeV}$ with the ATLAS detector, ATLAS-CONF-2020-026 [https://cds . cern.ch/record/2725727] (2020).

[6] ATLAS Collaboration, Measurements of gluon-gluon fusion and vector-boson fusion Higgs boson production cross-sections in the $H \rightarrow W W^{*} \rightarrow$ ev $\mu v$ decay channel in pp collisions at $\sqrt{s}=13 \mathrm{TeV}$ with the ATLAS detector, Phys. Lett. B 789 (2019) 508 arXiv: 1808.09054 [hep-ex].

[7] ATLAS Collaboration, Higgs boson production cross-section measurements and their EFT interpretation in the $4 \ell$ decay channel at $\sqrt{s}=13 \mathrm{TeV}$ with the ATLAS detector, Eur. Phys. J. C 80 (2020) 957 arXiv: 2004.03447 [hep-ex]. 